\title{
PLANETARY AND LUNAR EPHEMERIDES, LUNAR LASER RANGING, AND LUNAR PHYSICAL LIBRATIONS
}

\author{
X X NEWHALL, J. G. WILLIAMS, AND E. M. STANDISH, JR. \\ Jet Propulsion Laboratory \\ California Institute of Technology \\ Pasadena, California 91109-8099 USA
}

\section{The Planetary and Lunar Ephemerides}

The Jet Propulsion Laboratory (JPL) has recently produced a new integrated planetary and lunar ephemeris DE403/LE403. This ephemeris spans the interval JED 624912.5 (December 2, -3002, Julian) - JED 2817104.5 (November 14, 3000, Gregorian) and is an improvement on DE102 (Newhall et al., 1983) and DE200 (Standish, 1990). This integration carries the Cartesian states of the Sun, Moon, and planets, along with the three Euler angles describing the lunar physical librations.

\subsection{DYNAMICAL MODEL}

The dynamical model of the solar system consists of the following pointmass effects:

(1) The relativisitic $n$-body equations of motion (see Newhall et al., 1983) for the point-mass Sun, Moon, and planets;

(2) The three major asteroids Ceres, Pallas, and Vesta perturbing the Moon and planets; and

(3) The resultant accelerations on the Earth, Moon, and Mars due to 297 additional asteroids. (These accelerations are not computed simultaneously with the integration of the planets but are produced separately and read from a file.)

In addition, perturbations on the orbit of the Earth-Moon barycenter are included from the interaction of the point-mass Sun with the figure and solid-body tides of both the Earth and Moon; perturbations on the 
geocentric lunar orbit include effects of each of the Earth and Moon acting on the figure and solid tides of the other.

\subsection{NUMERICAL INTEGRATION}

The numerical integration of the solar-system bodies was done using the subroutine package DIVA, a variable-order, variable-step Adams method (Krogh, 1974). The planets, Moon, and lunar librations were integrated; the state of the Sun was computed from the relativistic conservation of center of mass. The step size was approximately 0.3 days; for DE403 there were 7.4 million steps.

The integration was done in nine successive sections. To preserve continuity and avoid noise and integration errors introduced by restarts at the beginning of each section, the integrator's difference tables were saved at the end of each integration section and then reloaded later to allow seamless continuation.

\subsection{EPHEMERIS REPRESENTATION}

The distributed ephemerides are in the form of Chebyshev polynomials. For the Sun, planets, and Moon the polynomials represent Cartesian position coordinates; they are solar-system barycentric for the Sun and planets and geocentric for the Moon. The lunar physical librations are expressed as three Euler angles defining a rotation between the Earth mean equator and equinox of J2000 and the lunar selenographic system. For a detailed description of Chebyshev representation of these ephemerides, see Newhall (1989).

Polynomial interpolation error is the difference between an interpolated value and the corresponding value obtained from the original integrator difference tables. For DE403, the maximum interpolation error is less than $0.5 \mathrm{~mm}$ for the Sun, Moon, and planets; for the libration angles the maximum interpolation error is less than $20 \mu$ as. A significantly compressed form of DE403, designated DE404, in which the interpolation-error criteria are relaxed, is available (Standish and Newhall, 1995). On that ephemeris, the maximum interpolation error is less than 25 meters for the Sun and planets and less than 1 meter for the Moon. The lunar librations are not included on DE404.

\section{Lunar Laser Ranging}

Since 1969, laser ranges (round-trip light travel times) to retroreflectors on the Moon have been acquired by terrestrial observatories. Beginning in early 1970 , ranges were sufficiently accurate for use in the determination of 
astronomical quantities. As more data were accumulated, estimates were made of the lunar ephemeris, the lunar physical librations, and, ultimately, parameters associated with General Relativity. This section contains a brief summary of the present status of lunar laser ranging (LLR); for a thorough review article, see Dickey et al. (1994).

\subsection{THE LLR DATA SET}

The present set of LLR data consists of more than 9900 normal points. From 1970 through 1983 the data are from only the McDonald Observatory of the University of Texas. Beginning in 1984, ranges were acquired at Mt. Haleakala on the island of Maui and then at CERGA in Grasse, France.

Uncertainties on the data were initially $20-30 \mathrm{~cm}$. In 1976 , instrumentation improvements yielded uncertainties of $10-15 \mathrm{~cm}$; subsequent continuing improvements have provided uncertainties in the $2-3 \mathrm{~cm}$ range since 1991 .

\subsection{THE OBSERVATION MODEL}

Computed ranges between each station and reflector are required for parameter estimation. Errors in the modeled values must be substantially less than the uncertainties of the ranges. The present model includes contributions from:

(1) Planetary and lunar ephemerides;

(2) UT1 and polar motion, including sub-daily effects in UT1;

(3) Geocentric station locations;

(4) Selenocentric reflector locations;

(5) Solid tides on the Earth and Moon, produced on each body by the gravity field of the other and by that of the Sun;

(6) Pole tides on the Earth, arising from the elastic deformation of the Earth caused by the displacement of the instantaneous pole from the mean pole;

(7) Precession and nutation of the Earth;

(8) Physical librations of the Moon;

(9) Atmospheric delay; and

(10) Relativistic effects, including the Lorentz contraction of the Earth and Moon, the time delay in the ranges caused by the combined potential of the Sun and Earth, and the transformation between TAI (atomic time) and coordinate time. 


\subsection{ESTIMATED PARAMETERS}

The data set permits the estimation of several parameters to which the observations are sensitive. In the estimation process, LLR data are combined with planetary data to allow the estimation of additional solar-system parameters. The solution set includes:

- Earth orbit initial conditions

- Lunar orbit initial conditions

- Lunar physical libration initial conditions

- The length of the astronomical unit

- $G M_{\text {Earth+Moon }}$

- $M_{\text {Earth }} / M_{\text {Moon }}$

- Station locations

- Reflector locations

- Lunar moments of inertia

- Lunar harmonics to third degree

- Lunar Love numbers $k_{2}, h_{2}$, and $l_{2}$

- Lunar dissipation time delay

- Earth dissipation time delays (and the consequent secular acceleration of the lunar longitude)

- Precession correction

- Long-period nutation terms

- Fast (sub-daily) UT1 terms

- Relativity parameters: $\beta, \gamma, \dot{G} / G, M_{\text {Gravitational }} / M_{\text {Inertial }}$ for the Earth, and geodetic precession

- UT1 and polar motion

The estimation of UT and polar motion treats those variables as stochastic parameters. A Kalman-filter approach is used to estimate a value of UT and the two components of polar motion at every data point. This process is done simultaneously with the estimation of a single (global) value of each of the remaining parameters in the set. For the single-station (McDonald) data before 1983, only the two parameters UT0 and variation of latitude are estimated. For data after that time, a priori covariances are sufficiently accurate to allow estimation of UT1 and both components of polar motion. 


\section{The Lunar Physical Librations}

\subsection{DEFINITION OF THE LIBRATIONS}

Lunar laser ranging (LLR) has enabled the determination of the lunar ephemeris and rotation history (physical librations) to a high degree of accuracy. The librations consist of a set of three Euler angles that define a rotation matrix between the Earth mean equator and equinox of J2000 and the selenographic system. The angles are $\phi$, the angle along the Earth's equator from the vernal equinox to the ascending node of the lunar equator; $\theta$, the inclination of the lunar equator to the Earth's equator; and $\psi$, the angle along the lunar equator from its node on the Earth's equator to the lunar prime meridian.

\subsection{DESCRIPTION OF THE LIBRATIONS}

There are two basic types of librations: longitude and latitude. The libration in longitude refers to the behavior of the combination $\Delta \psi+\Delta \phi \cos \theta$, the total variation of the angular position of the Moon about its axis. It is analogous to a variation in UT1 on Earth, except that the lunar libration has no known stochastic component.

The lunar libration in latitude refers to the variation of the position of the lunar pole in space (the combined effect of the two variations $\Delta \phi$ and $\Delta \theta)$ and is the equivalent of a combination of precession, nutation, and polar motion on Earth. The lunar north pole precesses one full revolution about the ecliptic pole every 18.6 years and is displaced from the ecliptic pole by $1.54^{\circ}$. By comparison, the Earth's north pole precesses one revolution about the ecliptic pole every 26,000 years and is displaced from the ecliptic pole by $23.4^{\circ}$. (For a thorough description of the lunar librations, see Eckhardt (1981).)

\subsection{FORCED VS. FREE LIBRATIONS}

Forced librations are those variations in lunar rotation and orientation caused by external time-varying torques originating from the gravitational effects of the Earth, Sun, and planets. Free librations are variations in rotation and orientation due to three natural libration modes and frequencies. They can be stimulated by impacts and internal processes. The free libration period is also a function of the lunar orbit period, which is increasing due to the effect of the Earth's tides. Passage of the libration period through a resonance with the external forcing terms can stimulate a free libration mode (Eckhardt, 1993).

The amplitude and phase of the free librations cannot be predicted; they must be measured. The free libration in longitude has a predicted period 
of 1056 days. The free libration in latitude has two predicted modes: a Chandler-like wobble with a period of about 74.6 years (when expressed in the rotating lunar frame), and a precession-like motion with a 79-year period (when expressed in the inertial frame).

\subsection{THE PHYSICAL LIBRATION MODEL}

The librations are the result of external torques on the Moon. Sources for torques include the point-mass Sun, Earth, Venus, and Jupiter interacting with the lunar figure; the effect of the point-mass Sun and Earth acting on the lunar solid tides; and the mutual interaction between the Earth and Moon figures.

Lunar elasticity and dissipation are modeled by assuming a time-varying moment of inertia $\mathbf{I}(t ; \tau)$ that is a function of the Earth-Moon vector $\mathbf{r}_{\mathrm{EM}}$ and lunar angular velocity $\omega$ at a retarded time $t-\tau$ :

$$
\mathbf{I}(t ; \tau)=f\left[\mathbf{r}_{\mathrm{EM}}(t-\tau), \omega(t-\tau)\right]
$$

The value of $\tau$ is typically 4 hours.

When the model is integrated backwards in time, the dissipative terms will contribute to an exponential growth in libration amplitude. Further, in a backwards integration the retarded-time formulation functions mathematically as an advanced-time equation and is inherently unstable, augmenting the exponential growth in amplitude.

The fundamental differential equation for the lunar rotation in terms of the lunar angular velocity $\omega$ is:

$$
\dot{\omega}(t)=\mathrm{I}(t ; \tau)^{-1}\left\{-\omega(t) \times \mathrm{I}(t ; \tau) \omega(t)-\dot{\mathrm{I}}(t ; \tau) \omega(t)+\mathrm{N}_{\text {pt-mass }}+\mathrm{N}_{\text {fig-fig }}\right\}
$$

where $\mathbf{I}(t ; \tau)$ is the inertia tensor defined above, $\mathbf{N}_{\text {pt-mass }}$ is the net torque resulting from point-mass effects, and $\mathbf{N}_{\text {fig-fig }}$ is the torque from the mutual interaction from the Earth and Moon figures.

When the above equation is integrated, the three libration angles $\phi, \theta$, and $\psi$ are obtained by integrating their corresponding differential equations:

$$
\begin{aligned}
\dot{\phi} & =\left(\omega_{x} \sin \psi+\omega_{y} \cos \psi\right) / \sin \theta \\
\dot{\theta} & =\omega_{x} \cos \psi-\omega_{y} \sin \psi \\
\dot{\psi} & =\omega_{z}-\dot{\phi} \cos \theta
\end{aligned}
$$

where $\omega_{x}, \omega_{y}$, and $\omega_{z}$ are the components of the angular velocity vector $\omega$ in the selenographic system.

\subsection{THE LIBRATION STUDY}

A large rotational dissipation was discovered from LLR analyses and is a clue about the lunar interior. Its origin is uncertain. Solid-body friction is an 
unlikely source; however, observations are consistent with viscous damping at the surface of a small fluid core interacting with the mantle (Yoder, 1981; Dickey et al., 1994).

\subsubsection{Motivation}

Free librations were reported by Calame (1977). The damping time should be geologically short, due to the small lunar $Q$ (about 26), and impacts appear statistically too infrequent to serve as the only source of excitation. Yoder (1981) shows that turbulence in a core-mantle boundary could function as a continuing, more frequent internal source of excitation and could account for the existence of free librations in the presence of strong dissipation. If free librations can be established, their presence would support the existence of a fluid core.

\subsubsection{Separation of Free and Forced Librations}

Previous researchers have compared observations with both an analytical theory and a numerical integration of forced librations. In this study a Fourier analysis approach is taken. First, initial conditions for the physical librations were derived from a fit to LLR data. Next, the librations were integrated simultaneously with the solar system bodies over the time span of interest. Then an iterative procedure was used for each of the three libration angles: (1) A Fourier analysis is done over the selected span (718 years $[262,144$ days] in this study) to identify the largest amplitudes; (2) these amplitudes are least-squares estimated; (3) the corresponding frequencies are then estimated; and (4) the resulting trigonometric series is evaluated and subtracted from the values of the input angles to provide the modified array of angle values to be Fourier analyzed. Iteration was continued until all components down to a selected amplitude level had been determined.

\subsection{FREE LIBRATION RESULTS}

In the case of longitude, an amplitude of $1.8^{\prime \prime}$ near the free libration period is seen; however, the free libration is blended with two forced amplitudes due to Venus (Eckhardt, 1982) having nearly identical periods of 1056.3 and 1056.4 days and resists separation. The free period cannot be determined precisely; if the two forced terms are subtracted out, the resulting free amplitude is strongly dependent on what free period is assumed. To illustrate this sensitivity, an assumed 1056.00-day period yields a free amplitude of $1.4^{\prime \prime}$; a 1056.20 -day period yields an amplitude of $0.9^{\prime \prime}$; and a 1056.28-day period gives an amplitude of $0.2^{\prime \prime}$.

The free libration in latitude exhibits a $3^{\prime \prime} \times 8^{\prime \prime}$ elliptical component in the pole oscillation with a 74.6-year period. There are no known nearby theoretical forced terms. 
The libration in latitude and the blend in longitude correspond to Calame's (1977) results.

It should be noted that the span of LLR data is 25 years. One might wonder how a 718-year analysis can be done on a support of only 25 years of data. The underlying assumption is that the physical model, data, and integration software are precise enough to permit extrapolation to the desired resolution. It is encouraging that the Fourier estimation-iteration procedure has yielded amplitudes and frequencies predicted by theory and that nothing unduly dramatic or spurious has appeared.

\section{Acknowledgement}

The research described in this paper was performed at the Jet Propulsion Laboratory, California Institute of Technology, under a contract with the National Aeronautics and Space Administration.

\section{References}

Calame, O. (1977) Free librations of the Moon from Lunar Laser Ranging, in Scientific Applications of Lunar Laser Ranging, J. D. Mulholland, ed., D. Reidel, Dordrecht, pp. $53-63$

Dickey, J. O., Bender, P. L., Faller, J. E., Newhall, X X, Ricklefs, R. L., Ries, J. G., Shelus, P. J., Veillet, C., Whipple, A. L., Wiant, J. R., Williams, J. G., and Yoder, C. F. (1994) Lunar Laser Ranging: A Continuing Legacy of the Apollo Program, Science, 265, pp. 482-490

Eckhardt, D. H. (1981) Theory of Libration of the Moon, The Moon and the Planets, 25, pp. 3-49

Eckhardt, D. H. (1982) Planetary and Earth Figure Perturbations in the Librations of the Moon, in High-Precision Earth Rotation and Earth-Moon Dynamics, O. Calame, ed., D. Reidel, Dordrecht, pp. 193-198

Eckhardt, D. H. (1993) Passing Through Resonance: The Excitation and Dissipation of the Lunar Free Libration in Longitude, Celest. Mech. Dyn. Astron., 57, pp. 307-324

Krogh, F. T. (1974) Changing Stepsize in the Integration of Differential Equations Using Modified Divided Differences, in Proceedings of the Conference of the Numerical Solution of Ordinary Differential Equations, Oct. 1972, Lecture Notes in Mathematics, 362, Springer-Verlag, New York, pp. 22-71

Newhall, X X, Standish, E. M., Jr., and Williams, J. G. (1983) DE102: a numerically integrated ephemeris of the Moon and planets spanning forty-four centuries, Astron. Astrophys., 125, pp. 150-167

Newhall, X X (1989) Numerical Representation of Planetary Ephemerides, Celest. Mech., 45, pp. 305-310

Standish, E. M., Jr. (1990) The observational basis for JPL's DE200, the planetary ephemerides of the Astronomical Almanac, Astron. Astrophys., 233, pp. 252-271

Standish, E. M., Jr. and Newhall, X X (1995) New Accuracy Levels for Solar System Ephemerides, in Proceedings of IAU Symposium 172: Dynamics, Ephemerides, and Astrometry of Solar System Bodies, Paris (this volume)

Yoder, C. F. (1981) The free librations of a dissipative Moon, Phil. Trans. R. Soc. Lond. Series A 303, pp. 327-338 